\title{
Fatores Ecológicos Associados à Colonização e aO DesenVolvimento de Macrófitas Aquáticas e Desafios de MANEJ $\mathbf{O}^{1}$
}

\author{
Ecological Factors Associated to Aquatic Macrophyte Colonization and Growth and \\ Management Challenges
}

THOMAZ, S.M. ${ }^{2}$

\begin{abstract}
RESUMO - As macrófitas constituem-se em uma importante assembléia de ecossistemas aquáticos continentais, mas seu crescimento excessivo pode provocar danos aos usos múltiplos de alguns ambientes. Durante o processo de sucessão ecológica, a maioria dos ecossistemas aquáticos é colonizada, em diferentes graus, pela vegetação aquática. No entanto, explosões populacionais são usualmente decorrentes de ações antrópicas, como introduções de espécies exóticas e alterações de habitats. O conhecimento da ecologia e biologia das espécies de macrófitas que colonizam ecossistemas tropicais ainda é escasso. Entretanto, esse conhecimento é fundamental para a predição do desenvolvimento da vegetação aquática e para subsidiar as medidas de manejo, quando estas forem necessárias. Os métodos de controle e manejo são eficazes em pequenos ambientes e sua aplicação pode ser acompanhada por uma série de impactos ecológicos, nem sempre avaliados apropriadamente. O desenvolvimento de métodos com reduzidos impactos ambientais e que sejam eficientes em grandes ecossistemas é um desafio. Deve-se ainda considerar que, embora em algumas situações o manejo seja necessário no sentido de reduzir uma parcela das populações de macrófitas, em outras ele deveria ser utilizado para estimular a colonização e o incremento desta vegetação.
\end{abstract}

Palavras-chave: ecossistemas aquáticos, reservatórios, fatores limitantes, sucessão ecológica.

\begin{abstract}
The aquatic macrophytes have been considered an important community in freshwater ecosystems. However, their excessive colonization and growth usually cause serious impacts on multiple use of these ecosystems. Most aquatic environments are colonized at different degrees by aquatic plants in some phase of ecological succession. Nevertheless, massive growth is usually associated with anthropogenic actions such as introduction of alien species and habitats of alterations. Knowledge about ecology and biology of the species that colonize tropical ecosystems is still scarce. This knowledge is fundamental to predict the development of aquatic vegetation and to subside its management, whenever necessary. The methods of control and management are efficient only in small ecosystems and their application is usually followed by several ecological impacts, not always well assessed. In general, the development of methods with reduced impacts and efficient in large ecosystems is a challenge. It is still important to consider that, although in some situations, management is necessary to reduce macrophyte populations, in others it should be used to stimulate the colonization and development of aquatic vegetation.
\end{abstract}

Key words: aquatic ecosystems, reservoirs, limiting factors, ecological succession.

Recebido para publicação em 12/11/2001 e na forma revisada em 17/12/2001.

Professor Adjunto - Universidade Estadual de Maringá/Nupélia, Av. Colombo 5790, 87020-900 Maringá-PR.

Planta Daninha, Viçosa-MG, v.20, p.21-33, 2002. Edição Especial 


\section{INTRODUÇÃO}

As macrófitas aquáticas colonizam, em diferentes graus, a maioria dos ecossistemas aquáticos lóticos e lênticos. Sua importância ecológica tem sido enfatizada por vários pesquisadores e está relacionada basicamente ao aumento da heterogeneidade espacial, que propicia a criação de habitats para macroinvertebrados (Esteves \& Camargo, 1986), aves (Voltz, 1995; Wallsten, 1998) e peixes (Delariva et al., 1994; Nakatani et al., 1997; Weaver et al., 1997), ao aumento da estabilidade da região litorânea e proteção das margens (Sand-Jensen, 1998) e, ainda, em determinadas circunstâncias, à retenção de nutrientes e poluentes (Gopal, 1987; Carpenter \& Lodge, 1986; Engelhardt \& Ritchie, 2001). Também, a biomassa das macrófitas pode representar, em determinados ecossistemas, a base de teias alimentares de herbivoria e detritivoria (Duarte et al., 1994; Esteves, 1998).

No entanto, as elevadas taxas de produção primária e o rápido crescimento populacional de várias espécies de macrófitas favorecem a colonização de vastas áreas, podendo afetar os usos múltiplos de ecossistemas aquáticos. Dentre os problemas mais comumente observados podem ser citados: redução da biodiversidade (Winton \& Clayton, 1996; Cilliers et al., 1996), prejuízos aos esportes náuticos (natação, esqui e pesca), entupimento de tubulações e canais de irrigação e, mais recentemente, prejuízos à produção de energia em usinas hidrelétricas (Mitchell et al., 1990; Itaipu Binacional, 1997; Thomaz \& Bini, 1999a, b). Assim, a despeito de sua importância ecológica, o termo "daninha" tem sido empregado com relativa freqüência na literatura, em referência a essa assembléia.

Em razão do exposto, os ecólogos aquáticos enfrentam, com freqüência, situações paradoxais, pois, ao mesmo tempo em que as macrófitas são reconhecidas como importantes do ponto de vista ecológico, podem representar um risco para a utilização dos recursos hídricos. A resolução desse paradoxo nem sempre é simples e os conflitos surgem quando se defrontam as opiniões, muitas vezes opostas, de ecólogos e técnicos ambientais. Em outras palavras, enquanto os primeiros enfatizam os benefícios ecológicos das macrófitas, os últimos quase sempre as encaram como prejudiciais ao aproveitamento múltiplo dos recursos hídricos. Assim, a avaliação do papel das macrófitas aquáticas em um determinado ecossistema depende necessariamente da obtenção e análise de dados de acordo com o método científico, com isenção de preconceitos e opiniões infundadas. Dentro dessa perspectiva, o conhecimento da ecologia das macrófitas aquáticas representa a chave para a tomada de decisões, e questões básicas ajudam a direcionar as ações no sentido de priorizar o "controle da vegetação aquática" ou "sua preservação".

No presente trabalho, pretende-se mostrar a importância de pesquisas básicas como suporte para o processo decisório sobre a utilização de medidas de controle e manejo de macrófitas aquáticas. Reconhecendo que, em algumas situações, o controle se torna necessário, alguns comentários sobre os principais métodos e os desafios de outros, ainda pouco utilizados, são também considerados.

\section{COLONIZAÇÃO E DESENVOLVIMENTO DA ASSEMBLÉIA DE MACRÓFITAS AQUÁTICAS}

Os ecossistemas aquáticos lênticos são reconhecidamente ambientes efêmeros do ponto de vista geológico. Embora o processo clássico de sucessão, que se inicia com um ambiente aquático oligotrófico e, após o assoreamento, culmina com um brejo ou uma floresta, tenha sido questionado com base em dados paleolimnológicos (Collinvaux, 1993), a sucessão ecológica se processa em todos os ecossistemas aquáticos lênticos. Na maioria dos casos, em alguma etapa desse processo, ocorre a colonização por macrófitas aquáticas.

Durante a sucessão, não ocorre somente alteração das espécies da assembléia de macrófitas aquáticas, mas há também substituição de grupos ecológicos ao longo do tempo. Esse fato é constatado especialmente quando essas mudanças ocorrem paralelamente ao incremento do estado trófico (eutrofização), o que leva à substituição de espécies submersas por emergentes e flutuantes (Esteves, 1998; Wetzel, 1983; Thomaz \& Bini, 1999a).

No caso de reservatórios, sistemas aquáticos que se tornaram comuns na paisagem, especialmente nas últimas décadas, as 
mudanças se processam mais rapidamente do que em lagos naturais. O envelhecimento precoce desses ecossistemas está associado à maior relação entre a área da bacia e a área alagada, fato que acelera o processo de assoreamento (Ford, 1990). A localização de reservatórios em bacias hidrográficas impactadas pelo desmatamento e pela atividade agrícola também contribui com esse processo. Como resultado direto do assoreamento, que reduz a profundidade das regiões litorâneas, extensos bancos de macrófitas podem se desenvolver nesses ecossistemas. Esse fato já vem sendo registrado nos grandes reservatórios brasileiros, a maioria dos quais construídos nas décadas de 60 e 70 (Itaipu Binacional, 1997).

Cabe ressaltar que, para uma mesma escala temporal, diferentes graus de colonização podem ser encontrados. A colonização depende, antes de tudo, de fontes de propágulos, que, através de mecanismos de dispersão, alcançam um novo ambiente. As principais fontes de dispersão das macrófitas são as aves aquáticas, os peixes, os próprios tributários e, mais recentemente, embarcações que transitam entre diferentes ecossistemas aquáticos.

O processo de dispersão pode ser considerado estocástico, mas o sucesso da colonização depende de uma multiplicidade de fatores ecológicos, muitos dos quais já conhecidos. Dentre os fatores físicos e químicos, podem ser citadas a estrutura e composição química do sedimento (Esteves \& Camargo, 1986; French \& Chambers, 1996), composição química da água (Feijoó et al., 1996; Bini et al., 1999), radiação subaquática (Spence, 1982; Rooney \& Kalff, 2000; Tavechio \& Thomaz, no prelo), velocidade da água (Carr et al., 1997), declividade da margem (Duarte \& Kalff, 1986; Bini, 2001) e exposição ao vento (Souza, 2000). Interações específicas, como a competição e herbivoria, também podem explicar o sucesso da colonização de espécies de macrófitas (Janes et al., 1996; Hootsmans, 1998; Sarbu \& Cristofor, 1998; Camargo \& Florentino, 2000).

Uma vez instaladas, o desenvolvimento da área colonizada pelas diferentes espécies pode seguir diferentes tendências (Figura 1). Quando as condições ambientais são desfavoráveis, como ocorre em ambientes aquáticos muito profundos, com elevada declividade das margens ou oligotróficos, a colonização por macrófitas aquáticas pode ser incipiente ou mesmo inexistente. Em outros ambientes, a área ocupada pela região litorânea pode se manter relativamente constante ao longo do tempo. Explosões populacionais logo após a formação de um ambiente aquático lêntico também podem ser registradas. Esse fato tem sido constatado após a construção de reservatórios, como foi o caso de Tucuruí (Brasil) e Kariba (África) (Tundisi, 1994; Mitchell et al., 1990). Essas explosões ocorrem em locais onde o "fetch" não é acentuado e estão associadas com os pulsos iniciais de nutrientes decorrentes da formação desses sistemas. Finalmente, a colonização por macrófitas pode ser máxima somente nos estágios mais avançados da sucessão, como se acredita que ocorra na maioria dos ecossistemas lênticos. Muitas dessas tendências apresentadas são teóricas e, certamente, uma gama de situações intermediárias, inclusive de regressão entre esses estágios (Collinvaux, 1993), pode ocorrer.

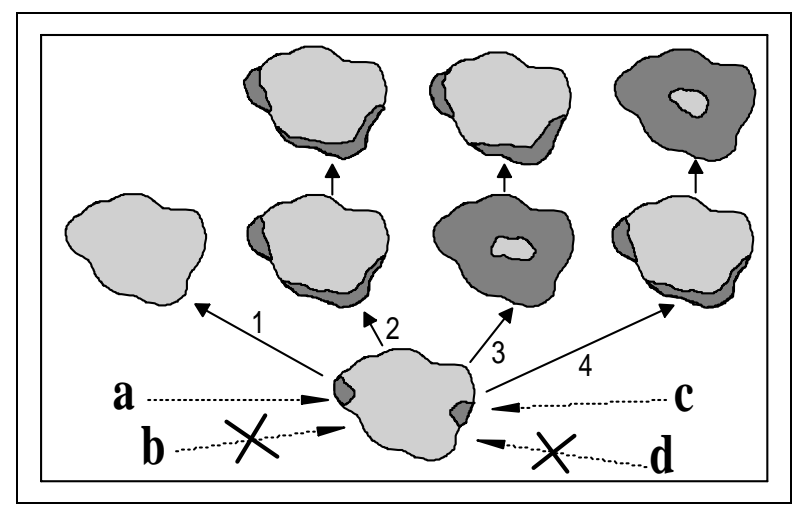

Figura 1 - Algumas possibilidades para a seqüência da colonização de ecossistemas aquáticos por macrófitas aquáticas. Algumas espécies, como b e d, pertencem ao "pool" regional, mas podem não alcançar o ecossistema em questão. Outras, como a e c, podem alcançar o ecossistema mas não colonizá-lo (situação 1), caso os fatores desfavoráveis prevaleçam. Alguns ecossistemas permanecem por longos períodos de tempo com uma relativa estabilidade das áreas colonizadas (situação 2), mas outros experimentam explosões populacionais logo após a chegada de uma espécie ou logo após a sua formação, como é o caso da construção de reservatórios (situação 3). A maioria dos ecossistemas passa por estágios de sucessão e somente após longos períodos de tempo apresentam-se largamente colonizados por macrófitas (situação 4). As áreas mais escuras correspondem às regiões colonizadas por macrófitas. 


\section{AÇÕES ANTRÓPICAS COMO CAUSAS DE EXPLOSÕES POPULACIONAIS DE MACRÓ- FITAS AQUÁTICAS}

Vários problemas decorrentes do desenvolvimento excessivo de macrófitas ocorrem após a interferência antrópica em um ecossistema. A introdução de espécies de macrófitas aquáticas é uma das interferências que geralmente vem acompanhada pelo crescimento indesejado desses vegetais. Os exemplos mais comuns referem-se às introduções do "aguapé" Eichhornia crassipes, uma espécie livre-flutuante, e da "elódea-brasileira" Egeria densa, submersa, ambas nativas da América do Sul. Atualmente essas espécies colonizam vários ecossistemas lênticos e lóticos nos Estados Unidos e em países da Europa, Ásia e Oceania, onde deslocaram várias espécies nativas de macrófitas (Mitchell et al., 1990; Mansor, 1996; Clayton, 1998; Pieterse, 1998; Dutartre et al., 1999). Hipoteticamente, o desenvolvimento das espécies tropicais introduzidas em países de regiões temperadas tende a se tornar cada vez mais acentuado, como resultado direto do efeito estufa. Em outras palavras, os limites da distribuição geográfica dessas espécies, introduzidas no hemisfério norte, devem se estender para latitudes mais elevadas, em decorrência do aquecimento global.

As introduções acidentais ou intencionais têm causado preocupação aos órgãos ambientais de vários países. Mecanismos eficientes para evitar as introduções ainda são um grande desafio. A confecção e distribuição de cartões com fotos e características das espécies-alvo, alertando sobre os problemas decorrentes de sua introdução, constituem uma medida atenuante em áreas potencialmente colonizáveis. Acredita-se que a disponibilização dessas informações para o público que utiliza os recursos hídricos retarde a instalação de várias espécies daninhas.

Outros tipos de interferência antrópica que podem estimular o desenvolvimento indesejado de macrófitas aquáticas relacionam-se à manipulação de habitats. Como exemplos podem ser citadas a construção de reservatórios, a manipulação dos niveis hidrométricos e a própria eutrofização artificial.

A criação de reservatórios altera substancialmente os ecossistemas, adicionando, ainda, novas áreas para a colonização por organismos aquáticos. A criação desses ambientes afeta de diferentes maneiras o desenvolvimento e a riqueza das assembléias de macrófitas aquáticas (Rørslett \& Johansen, 1996; Thomaz \& Bini, 1999a, b; Thomaz et al., 1999). Os fatores que afetam a colonização de reservatórios por macrófitas relacionam-se: à estrutura dos habitats, cuja heterogeneidade pode ser reduzida ou incrementada após a formação do novo ambiente; ao padrão de flutuação dos níveis de água imposto pelo reservatório; à velocidade da água, menor nestes ambientes; e à sedimentação, mais acentuada nos reservatórios, que resulta em maior penetração de luz e perda de nutrientes da coluna de água. Esses eventos são sumarizados na Figura 2. Assim, além dos processos estocásticos, relacionados com a dispersão, a estrutura das assembléias e o grau de colonização de novos ambientes aquáticos são resultantes da combinação entre os fatores mencionados. Algumas possibilidades decorrentes dos efeitos desses fatores sobre a vegetação aquática são apresentadas em Thomaz \& Bini (1999b).

O rápido incremento de nutrientes e sólidos suspensos nos ecossistemas aquáticos, resultantes da ação antrópica (eutrofização artificial), pode ser considerado um dos processos que mais afetam a estrutura das assembléias de macrófitas aquáticas. A evolução do processo de eutrofização causa a perda de espécies submersas, pois, com o desenvolvimento maciço de algas planctônicas, a radiação subaquática passa a ser o principal fator limitante (Kraulec \& Kaplan, 1994; Królikowska, 1997; Sarbu \& Cristofor, 1998); porém, nem sempre os ecossistemas aquáticos eutróficos são desprovidos de vegetação submersa. De fato, tem sido constatado que vários ambientes aquáticos eutróficos são mantidos com elevada transparência da água e baixas densidades de fitoplâncton, pela presença maciça de macrófitas aquáticas submersas. Mecanismos cascata (p. ex., predação do fitoplâncton pelo zooplâncton que utiliza a região litorânea como refúgio), competição por nutrientes com o fitoplâncton e ação de substâncias alelopáticas são alguns dos mecanismos que explicam a presença de estandes bem desenvolvidos de macrófitas submersas em ecossistemas eutróficos (Coops \& Doef, 1996; Jeppesen et al., 1997; Meijer \& Hosper, 1997; Stansfield et al., 1997). Assim, dois estágios alternativos relativamente estáveis 
têm sido reconhecidos em ecossistemas aquáticos eutróficos: um com elevada turbidez biogênica, decorrente da presença maciça do fitoplâncton, e outro com águas transparentes e presença de macrófitas submersas (Sheffer et al., 1993; Meijer \& Hosper, 1997).

Em determinadas situações, o incremento de nutrientes na água estimula a colonização

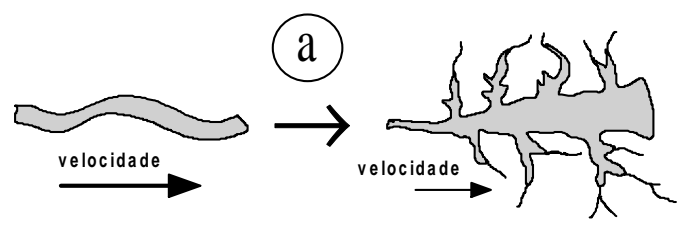

REDUÇĀO DA VELOCIDADE DA ÁGUAE AUMENTO DO DESENVOLVIMENTO DE MARGEM $\downarrow$

FAVORECEM A COLONIZAÇÃO E O AUMENTO DA RIQUEZA DE ESPÉCIES DA ASSEMBLÉIA DE MACRÓFITAS AQUÁTICAS

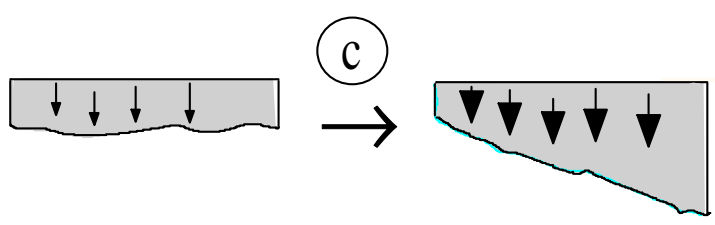

AUMENTO DAS TAXAS DE SEDIMENT AÇAO $\downarrow$

ELEVA A TRANSPARÊNCIA DA ÁGUA E A RETENÇÃO DE MATERIAL EM SUSPENSÃO E NUTRIENTES, FAVORECENDO O DESENVOLVIMENTO DE MACRÓFIT AS SUBMERSAS por espécies de macrófitas aquáticas livreflutuantes, que extraem os nutrientes da coluna de água. Esse fato é comum no início da formação de reservatórios, como já considerado anteriormente, ou após procedimentos operacionais que promovem alterações dos níveis de água nesses ambientes, que também podem resultar em incrementos das concentrações de nutrientes.
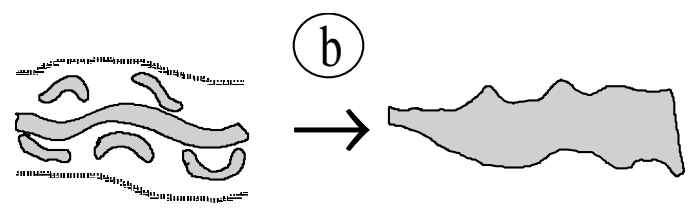

REDUÇĀO DA DIVERSIDADE DE HÄBT ATS

FAVORECE A REDUÇÃO DA RIQUEZA DE ESPÉCIES DE MACRÓFITAS AQUÁTICAS

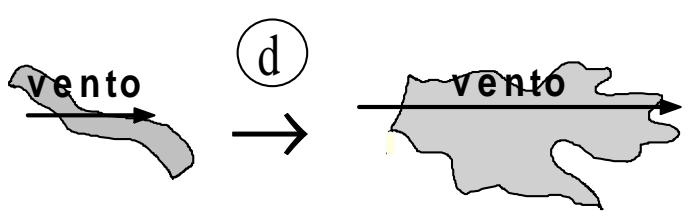

AUMENTO DA EXPOSIÇĀO AO VENTO ("FETCH")

$\downarrow$

ALTERA OS PADRÕES DE CIRCULACCÃO DAS MASSAS DE ÁGUA E AUMENT A O ESTRESSE SOBRE A ASSEMBLÉIA DE MACRÓFITAS
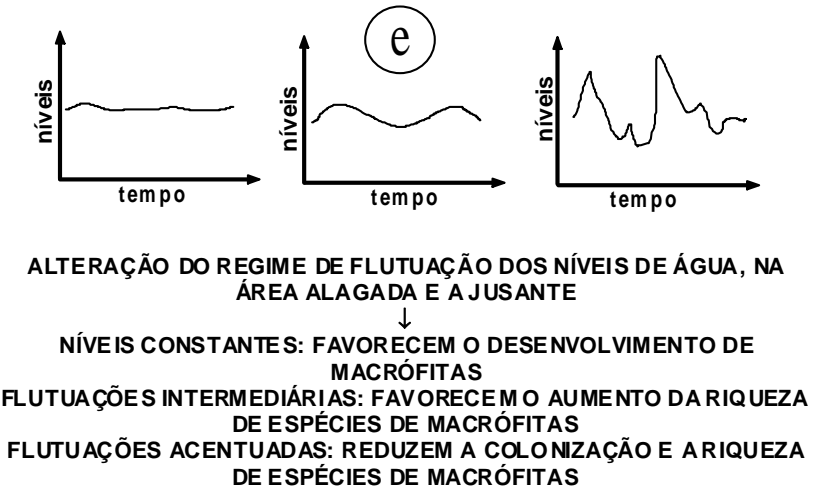

Figura 2 - Algumas transformações decorrentes da formação de reservatórios e seus efeitos sobre as assembléias de macrófitas aquáticas. (a) transformação de um rio encaixado em reservatório; (b) transformação de um rio de planície de inundação em reservatório; (c) alteração das taxas de sedimentação; (d) comparação dos efeitos do vento em um rio e em um reservatório; (e) possíveis transformações do regime hidrológico após a formação de reservatórios. 
Cabe ressaltar que no Brasil, contrariamente ao que se observa em países da Europa e da América do Norte, os problemas atualmente registrados com o crescimento de macrófitas são provocados por espécies nativas, como Egeria najas e E. densa (submersas) e Eichhornia crassipes, Pistia stratiotes e Salvinia auriculata (livre-flutuantes). Os reservatórios, em particular, têm sido os ecossistemas mais afetados pelo desenvolvimento maciço dessas espécies, fato que geralmente não é observado em seus habitats naturais, as áreas alagáveis. Esses ambientes são submetidos anualmente aos pulsos de inundação, que funcionam como um mecanismo natural de controle, reduzindo a biomassa de macrófitas acumulada em períodos mais favoráveis (Bonetto, 1975).

\section{ALGUNS DESAFIOS QUE PRECEDEM O MANEJ O DAS MACRÓFITAS AQUÁTICAS}

A previsão da ocorrência de problemas com macrófitas aquáticas, suas causas e a possível dimensão encontram-se entre os desafios que devem ser defrontados antes que medidas de controle ou manejo se façam necessárias. Essa posição preventiva, no sentido de "anteceder os problemas ao invés de constatá-los e utilizar tratamentos a posteriori", tem sido considerada uma mudança fundamental de paradigma que pode fortalecer a ecologia enquanto ciência (Rigler \& Peters, 1995).

Segundo Thomaz \& Bini (1999a), deve-se questionar, diante de um problema em potencial com macrófitas aquáticas, se realmente o manejo ou controle se faz necessário e, ainda, caso o seja, em que grau este deve ser empregado. De fato, do ponto de vista ecológico, o enfoque sugerido por vários técnicos ambientais, de que "quanto mais cedo o controle for empregado, mais efetivo este será”, tem pouca utilidade (Thomaz \& Bini, 1999a).

Alguns exemplos têm demonstrado que a colonização por várias espécies restringe-se a áreas reduzidas, assim permanecendo durante longo período de tempo. Caso se reconheça de antemão que o crescimento é inibido por fatores limitantes que tendem a permanecer no ecossistema em questão, então medidas de controle são desnecessárias, o que é altamente vantajoso em termos ecológicos e econômicos. Além disso, tem sido demonstrado que a colonização moderada por macrófitas aquáticas é altamente desejável, pois aumenta a biodiversidade local das assembléias de peixes (Dibble et al., 1996; Agostinho, A. A. - comunicação pessoal). Essa é a situação da margem brasileira do reservatório de Itaipu, onde os bancos de E. najas restringem-se a profundidades inferiores a 2,5 metros (Thomaz et al., 1998). A elevada declividade das margens desse reservatório, sua grande profundidade e a reduzida penetração de radiação subaquática impedem o avanço das áreas colonizadas. De fato, o controle desses pequenos bancos não é recomendado, pois a tendência é de que se mantenham com o atual grau de colonização enquanto as condições morfométricas e limnológicas do reservatório permanecerem como tal (Thomaz et al., 1999). Contrariamente, em função da amplitude espacial, o controle certamente representaria um grande dispêndio econômico e não eximiria o reservatório de novas colonizações.

As considerações observadas anteriormente apontam para a importância central dos levantamentos e monitoramentos para direcionar as ações a serem tomadas quanto às assembléias de macrófitas aquáticas. Quando bem elaborados, os resultados dos levantamentos e monitoramentos podem responder a importantes questões, como:

i. Que espécies colonizam o ecossistema de interesse e com que freqüência elas ocorrem? Qual o grau de colonização (em termos de área e profundidade de ocorrência)?

ii. Quais são as "daninhas" em potencial? Algumas já provocam prejuízos a algum tipo de uso do recurso hídrico em questão?

iii. Quais são os locais mais colonizados? Quais são os possiveis fatores limitantes, identificados através de relações empíricas entre a presença, biomassa ou área colonizada e variáveis morfométricas e limnológicas, obtidas durante o levantamento?

No entanto, os levantamentos e o monitoramento não bastam. Os estudos ecológicos básicos ainda são escassos, especialmente em regiões tropicais, e também deveriam preceder qualquer medida efetiva de controle ou manejo. Estes estudos podem envolver amostragens em vários ecossistemas ou em várias estações de coleta de um mesmo ecossistema, e os resultados podem ser utilizados na elaboração de 
modelos preditivos. Não menos útil é o conhecimento sobre biologia e ecologia das espécies, obtido diretamente em campo ou em experimentos que testam os efeitos de prováveis fatores limitantes, identificados em campo durante as fases de levantamento e monitoramento, sobre o crescimento das macrófitas aquáticas.

O enfoque preditivo, por exemplo, fornece modelos quantitativos que podem ser aplicados com sucesso no manejo de ecossistemas aquáticos (Hakanson \& Peters, 1995; Rigler \& Peters, 1995). Esse enfoque tem sido utilizado para a predição de diferentes parâmetros (por exemplo, concentrações de fósforo ou clorofila- $a$ ), processos (por exemplo, produção primária e taxas de respiração) ou biomassa e potencial de colonização de diferentes espécies aquáticas e terrestres.

Três exemplos com macrófitas aquáticas serão utilizados para ilustrar esse enfoque. Duarte et al. (1986) e Canfield Jr. \& Duarte (1988) desenvolveram vários modelos para predizer a área colonizada por macrófitas aquáticas em lagos da América do Norte. Os modelos com maior capacidade de predição da cobertura total de macrófitas para lagos da Flórida (EUA), por exemplo, basearam-se em variáveis simples, como tamanho dos lagos, radiação anual da superfície do lago e radiação média anual na profundidade média do lago.

Um segundo modelo, ainda mais simples, baseado nos resultados obtidos em vários lagos da América do Norte, também foi desenvolvido por Duarte \& Kalff (1986), que obtiveram a seguinte relação entre a biomassa máxima de macrófitas submersas (BM, em $\mathrm{gPF} \mathrm{m}^{-2}$ ) e a declividade do sedimento (dec, em \%):

$\mathrm{BM}=-28,9+1,403 \mathrm{dec}^{-0,81}$, se dec $<5,33 \%$

$\mathrm{BM}=13,2+3,434 \mathrm{dec}^{-0,81}$, se dec $>5,33 \%$

Esse modelo explicou $72 \%\left(R^{2}\right)$ dos resultados da biomassa máxima de macrófitas submersas, mas o grau de predição chegou a 88\% quando as concentrações de matéria orgânica do sedimento foram a ele incorporadas.

O terceiro exemplo refere-se a um modelo gerado com dados obtidos no reservatório de Itaipu com a macrófita submersa Egeria najas. Quando os valores da biomassa desta espécie são plotados contra o coeficiente de atenuação da radiação subaquática, os pontos assumem a forma de um envelope, cujos limites superiores da biomassa podem ser preditos através de uma regressão linear simples (Figura 3). A utilização de outras variáveis, como as concentrações de fósforo e nitrogênio da água e do sedimento e a turbidez, em modelos de regressão múltipla elaborados para explicar a biomassa de E. najas neste reservatório confirmaram a importância da radiação subaquática, visto que apenas a turbidez apresentou coeficiente parcial significativo (Bini, 2001). Esses resultados têm um significado direto para as estratégias de manejo. Suponha que o controle da biomassa das espécies de macrófitas submersas nesse ambiente seja necessário. Nesse caso, as estratégias dificilmente alcançariam sucesso somente pela redução dos aportes de nutrientes, muito embora essa estratégia talvez fosse adequada para a redução da biomassa das espécies livre-flutuantes, cuja distribuição encontra-se melhor relacionada com as concentrações de nitrogênio e fósforo da água (Bini et al., 1999).

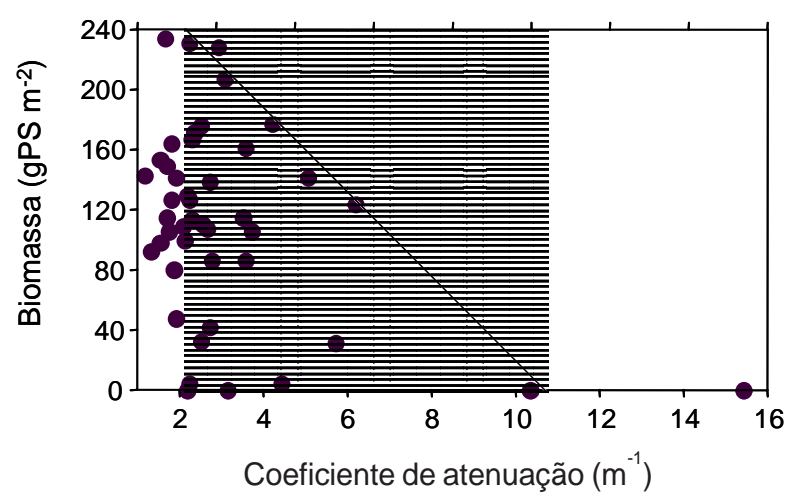

Figura 3 - Relação entre a biomassa de E. najas em braços do reservatório de Itaipu e o coeficiente de atenuação da radiação. Os valores máximos de biomassa podem ser preditos pela linha pontilhada (Bini, 2001).

Os exemplos anteriores demonstram que a utilização da abordagem preditiva auxilia sobremaneira na elaboração das estratégias de manejo. Em outras palavras, no caso específico das macrófitas aquáticas, através de variáveis morfométricas ou abióticas da água e do sedimento, de fácil obtenção, essa abordagem permite, entre outras, as seguintes possibilidades: 
- predizer quais locais estão mais propícios a serem colonizados, o que permite direcionar as medidas preventivas para esses locais;

- confeccionar mapas de risco, que demonstram os prováveis cenários decorrentes de alterações das variáveis independentes; e

- indicar, com base nos resultados de campo, quais fatores devem ser controlados para que o manejo efetivo da vegetação aquática seja alcançado.

Embora importantes para o manejo, os modelos preditivos, na maior parte das vezes, apenas indicam possíveis relações causais. Assim, estudos básicos de ecologia ou biologia, desenvolvidos em campo ou que utilizam experimentos em microcosmos, mesocosmos ou mesmo em ecossistemas inteiros ("whole ecosystem experiments"), complementam os estudos preditivos e também auxiliam na elaboração de estratégias de manejo.

Em estudos sobre a produção de órgãos vegetativos de Potamogeton illinoensis, realizados em canais de irrigação do sul da Argentina, Bezic et al. (1996) concluíram que o uso de controle mecânico para destruir alta proporção de rizomas do tipo I, antes que os rizomas do tipo II se desenvolvam, poderia ser efetivo para reduzir a sobrevivência de populações desta espécie na estação sazonal subseqüente. Alternativamente, a introdução de medidas de controle para reduzir o suplemento de produtos da fotossíntese para a formação dos rizomas (através da destruição de folhagem, por exemplo), durante o período que precedesse a formação dos rizomas do tipo II, teria o mesmo efeito. Assim, este exemplo deixa claro que medidas de manejo efetivas para $P$. illinoensis pressupõem um conhecimento básico de biologia dessa espécie: em que período do ano são formados os diferentes tipos de rizoma.

O segundo exemplo refere-se à estimativa de taxas fotossintéticas de espécies submersas em função da intensidade de radiação subaquática, realizada experimentalmente. Experimentos com esse enfoque têm sido empregados por vários pesquisadores (Haramoto \& Ikusima, 1988; Hootsmans et al., 1996; Tavechio \& Thomaz, no prelo) e fornecem uma resposta básica para uma importante questão que surge quando o manejo se faz necessário: qual o limite de radiação para que a produção primária líquida seja maior que zero? Em outras palavras, qual a intensidade máxima de radiação subaquática que ainda permite o crescimento da espécie em questão? Os dados resultantes são prontamente aplicados em campo para predizer os locais mais afetados e a distribuição de espécies submersas ou os níveis de redução da radiação subaquática necessários para atingir o controle efetivo. Esse enfoque, que vem sendo aplicado no reservatório de Itaipu (Tavechio \& Thomaz, no prelo), fornece, juntamente com resultados obtidos em campo, subsídios para a confecção de mapas de risco da ocorrência de $E$. najas neste reservatório.

Em suma, os estudos de biologia e ecologia básica de macrófitas aquáticas que utilizam os enfoques preditivo e experimental ainda são escassos em ecossistemas aquáticos tropicais. Poucas espécies têm recebido muita atenção, destacando-se dentre elas o aguapé Eichhornia crassipes, a alface-d'água Pistia stratiotes e várias espécies da Pteridófita Salvinia spp., todas livre-flutuantes, que têm provocado sérios danos aos usos múltiplos de diferentes ecossistemas aquáticos. Dentre as submersas, os estudos se concentraram também em espécies que prejudicam os usos múltiplos, como a elódea-brasileira Egeria densa e Hydrilla verticilata, esta última ainda não registrada no Brasil. É interessante ressaltar que mesmo para espécies nativas da América do Sul, como E. densa, original das bacias do ParaguaiParaná, a maioria dos estudos de ecologia tem sido desenvolvida em regiões temperadas (Haramoto \& Ikusima, 1988; Clayton, 1998). Contrariamente, aspectos ecológicos de várias espécies que potencialmente podem causar prejuízos aos usos múltiplos de ecossistemas aquáticos tropicais permanecem praticamente desconhecidos.

Assim, o desconhecimento de aspectos biológicos e ecológicos básicos de várias espécies de macrófitas aquáticas tropicais pode comprometer o manejo, caso este seja necessário a curto prazo. O desenvolvimento desses estudos pode ser considerado um importante desafio para o manejo efetivo da vegetação aquática, quando este se faz necessário. 


\section{DESAFIOS DA APLICAÇÃO DO MANEJ O ÀS MACRÓFITAS AQUÁTICAS}

A partir de certo limite, o desenvolvimento da vegetação aquática pode passar a ser considerado prejudicial aos usos múltiplos de ecossistemas aquáticos e, então, os métodos de controle ou manejo são necessários. Esses métodos usualmente são colocados em três categorias, mostradas no Tabela 1.

Tabela 1 - Categorias e exemplos de métodos utilizados no manejo e controle de macrófitas aquáticas (segundo Pieterse \& Murphy, 1990)

\begin{tabular}{|l|l|}
\hline \multicolumn{1}{|c|}{ Métodos } & \multicolumn{1}{c|}{ Exemplos } \\
\hline Físicos/mecânicos & $\begin{array}{l}\text { Remoção manual, manipulação dos } \\
\text { níveis de água, controle da vazão, } \\
\text { uso de dragas, aumento da turbidez } \\
\text { da água e cobertura do sedimento } \\
\text { com diversos tipos de materiais }\end{array}$ \\
\hline Químicos & Herbicidas \\
\hline Biológicos & $\begin{array}{l}\text { Utilização de predadores (peixes e } \\
\text { insetos), competidores, parasitas e } \\
\text { substâncias alelopáticas }\end{array}$ \\
\hline
\end{tabular}

Os métodos mencionados vêm sendo aplicados com sucesso em vários ecossistemas aquáticos de reduzido tamanho, mas sua utilização em grandes ambientes ainda permanece um desafio. Em seguida, serão consideradas, de forma resumida, algumas particularidades e alguns desafios específicos de cada uma das categorias apresentadas.

Os métodos físicos ou mecânicos são os que apresentam a vantagem de não-utilização de produtos ou organismos nocivos ou que persistem no ecossistema após sua introdução. Dentre os métodos físicos, a manipulação dos níveis de água pode ser considerado o único com potencial para ser utilizado em grande escala, pois a totalidade da região litorânea poderia ser atingida simultaneamente com essa manipulação. Dessa forma, sua utilização fica restrita a ecossistemas que permitem esse tipo de interferência, como os reservatórios, por exemplo. A despeito de terem sido utilizados com sucesso em alguns reservatórios de região temperada, poucos exemplos podem ser citados nos trópicos. No reservatório de Paulo Afonso (rio São Francisco, BA), uma combinação entre o controle dos níveis de água (aumento da vazão) e a retirada de grandes bancos de Egeria densa do sedimento alcançou relativo sucesso no manejo desta espécie (Itaipu Binacional, 1997). No entanto, nem sempre o manejo é efetivo utilizando-se este método e, mesmo que o seja, a manipulação dos níveis de água para o manejo deve ser compativel com a manipulação voltada para a operação do ambiente em questão (produção de energia em reservatórios, por exemplo).

Alguns problemas gerais envolvem os métodos físicos ou mecânicos. Podem ser citados, entre eles, a disposição dos detritos removidos, a deterioração da qualidade da água quando os detritos permanecem no ambiente e a dispersão de propágulos para outras regiões.

A remoção manual, um dos métodos mecânicos mais simples, tem sido utilizada com sucesso em pequena escala em praias do reservatório de Itaipu, como a do município de Santa Helena (PR), onde a macrófita submersa E. najas tem prejudicado localmente as práticas de lazer (natação, pesca e esportes náuticos). O manejo é realizado usualmente por pescadores ou ex-pescadores, que podem receber salários maiores com esta prática do que com a própria pesca.

Os métodos químicos são os menos aceitos pela sociedade, por utilizarem substâncias cuja toxicidade a diferentes espécies e ao próprio homem nem sempre é inteiramente conhecida. Essa é uma da causas à qual pode ser atribuído o decréscimo do número de estudos que enfocam a utilização de herbicidas no manejo e controle da vegetação aquática, registrado, por exemplo, nos congressos da "Sociedade Européia de Pesquisas em Daninhas Aquáticas" (Caffrey $\&$ Wade, 1996). Ainda que o uso de herbicidas específicos para ambientes aquáticos seja permitido mesmo em países desenvolvidos (EUA, por exemplo), sua aplicação é motivo de controvérsia em vários países europeus. Ainda não foi irrefutavelmente comprovado, por exemplo, que a exposição a longo prazo a alguns herbicidas, como é o caso do 2,4-D, utilizado no combate ao aguapé, seja inofensiva para o ambiente e para a saúde humana (Pieterse, 1998). Pelo contrário, efeitos crônicos associados à exposição a esse composto já foram 
registrados em invertebrados e peixes (Murphy \& Barret, 1990). Assim, a utilização de substâncias com o maior grau de especificidade possível, ou seja, que atinjam somente a espéciealvo, constitui-se em grande desafio para a aplicação de métodos químicos.

A utilização de herbicidas diretamente no ambiente aquático deve ser precedida de uma série de medidas, que incluem testes de toxicidade, envolvendo organismos nativos da região onde os herbicidas vão ser aplicados, e acompanhada pelo monitoramento dos possíveis impactos no ecossistema. A análise da biota não deve se restringir aos efeitos agudos, mas efeitos crônicos também devem ser considerados. Murphy \& Barret (1990) sugerem o seguinte protocolo para o emprego de herbicidas em ambientes aquáticos: delineamento preliminar dos efeitos; testes detalhados de toxicidade, envolvendo os efeitos agudos; testes dos efeitos crônicos; estudos de bioacumulação; e avaliação ambiental.

O controle biológico tem aparecido como uma alternativa à aplicação de métodos químicos. Vários tipos de organismos, como fungos (Barreto et al., 2000), insetos (Harley \& Forno, 1990) e peixes (Pieterse \& Murphy, 1990), podem ser aplicados com esse intuito. Um dos problemas com esse método é que, na maioria das vezes, utilizam-se espécies exóticas, que podem trazer severos prejuízos ambientais. Outro ponto negativo é que, uma vez instalada no ecossistema, dificilmente se consegue retirar a espécie introduzida. Um dos exemplos mais comuns no controle biológico de plantas aquáticas submersas é a utilização da carpa-capim. A literatura científica é contraditória quando aborda a ação dos peixes herbívoros no controle de macrófitas aquáticas e sua capacidade de causar alterações profundas no ambiente (Agostinho \& Júlio Jr, 1996; Delariva \& Agostinho, 1999). Segundo esses autores, os impactos potenciais de peixes herbívoros estão ligados à redução dos locais de postura de algumas espécies, dos substratos que desenvolvem organismos que servem como alimento de outras e dos abrigos de espécies pequenas e formas juvenis das maiores, além da introdução de patógenos e parasitas que afetam as espécies nativas. Essas alterações contribuem para a redução da biodiversidade de locais colonizados por espécies introduzidas.
Um dos grandes desafios da aplicação de métodos biológicos de controle é a utilização de espécies nativas. Como exemplo, pode-se considerar Pterodoras granulosus, uma espécie de peixe omnivora, nativa da bacia do rio Paraná, cujos conteúdos estomacais contêm vegetação submersa em elevada freqüência (Hahn et al., 1992). O uso de substâncias alelopáticas que tenham elevada especificidade e de fungos, que aparentemente apresentam alta especificidade, também pode ser considerado estratégia promissora no manejo da vegetação aquática.

De forma geral, sabe-se que as macrófitas aquáticas apresentam múltiplas e complexas funções nos ecossistemas aquáticos, especialmente nos de menor profundidade. Do ponto de vista de aproveitamento dos recursos hídricos pelo ser humano, os conflitos no manejo surgem porque são reconhecidos "benefícios" e "prejuízos", cuja interpretação depende dos interesses individuais e de grupo de quem está envolvido com a situação, do grau e tipo de aproveitamento do ecossistema e, até mesmo, da formação de quem está efetuando a análise. Como já salientado no início deste texto, basta relembrar os conflitos entre técnicos ambientais e ecólogos, por exemplo. Uma situação ainda mais extrema envolveria, sem dúvida, o confronto entre as opiniões de técnicos ambientais e ambientalistas.

O grande desafio, então, consiste em empregar o manejo voltado para a manutenção da função ecológica das macrófitas aquáticas e não utilizá-lo buscando simplesmente a eliminação das populações, como ele é usualmente empregado. Tendo em vista o importante papel ecológico das macrófitas aquáticas, uma parcela de suas populações deve sempre ser mantida no sentido de preservar os "benefícios ecológicos" decorrentes de sua presença. Dessa forma, muito embora em algumas situações o manejo seja realmente necessário no sentido de reduzir uma parcela das populações de macrófitas, em outras este deveria ser utilizado para estimular a colonização e o incremento desta vegetação.

\section{AGRADECIMENTOS}

À leitura crítica do manuscrito e às sugestões da Dra. Rosemara Fugi (Universidade 
Estadual de Maringá) e do Dr. Luis Maurício Bini (Universidade Federal de Goiânia).

\section{LITERATURA CITADA}

AGOSTINHO, A. A.; JULIO Jr., H. F. Ameaça ecológica: peixes de outras águas. Ci. Hoje, v. 2, n. 4, p. 36-44, 1996.

BARRETO, R. et al. Biological control of neotropical aquatic weeds with fungi. Crop Protec., v. 19, p. 697-703, 2000 .

BEZIC, C.; DALL'ARMELLINA, A.; GAJARDO, O. Studies on vegetative production of Potamogeton illinoensis Morong in southern Argentina. Hydrobiologia, v. 340, p. 7-10, 1996.

BINI, L. M. Dinâmica populacional de Egeria najas Planchon (Hydrocharitaceae): sobrevivência de uma espécie submersa em um ambiente subtropical com elevada turbidez (reservatório de Itaipu Binacional, Brasil Paraguai). Maringá: Universidade Estadual de Maringá, 2001. 134 p. Tese (Doutorado em Ecologia) Universidade Estadual de Maringá, 2001.

BINI, L. M. et al. Aquatic macrophyte distribution in relation to water and sediment conditions in the Itaipu Reservoir, Brazil. Hydrobiologia, v. 415, p. 147-154, 1999.

BONETTO, A. A. Hydrologic regime of the Paraná River and its influence on ecosystems. In: HASLER, A. D. Coupling of land and water systems. New York, Springer Verlag, 1975. p. 175-197.

CAMARGO, A. F. M.; FLORENTINO, E. R. Population dynamics and net primary production of the aquatic macrophyte Nymphaea rudgeana C. F. Mey in a lotic environment of the Itanhaém River basin (SP, Brazil). R. Bras. Biol., v. 60, n. 1, p. 83-92, 2000.

CAFFREY, J. M.; WADE, P. M. The european weed research society and the management and ecology of freshwater plants. Hydrobiologia, v. 340, p. 9-13, 1996.

CANFIELD Jr., D. E.; DUARTE, C. M. Patterns in biomass and cover of aquatic macrophytes in lakes: a test with Florida lakes. Can. J. Fish. Aquatic. Sci., v. 45, p. 19761982, 1988.

CARPENTER, S. R.; LODGE, D. M. Effects of submersed macrophytes on ecosystem processes. Aquatic. Bot., v. 26, p. 341-370, 1986.

CARR, G. M.; DUTHIE, H. C.; TAYLOR, W. D. Models of aquatic plant productivity: a review of the factors that influence growth. Aquatic. Bot., v. 59, p. 195-215, 1997.
CILLIERS, C. J.; ZELLER, D.; STRYDOM, G. Short- and log-term control of water lettuce (Pistia stratiotes) on seasonal water bodies and on a river system in the Kruger National Park, South Africa. Hydrobiologia, v. 340, p. 173-179, 1996.

CLAYTON, J. Aquatic plant management in New Zealand: achievements and directions. In: MONTEIRO, A.; VASCONCELOS, T.; CATARINO, L. Management and ecology of aquatic plants. In: EWRS INTERNATIONAL SYMPOSIUM ON AQUATIC WEEDS, 10, 1998, Lisbon. Proceedings... Lisbon: APRH, 1998. p. 335-340.

COLLINVAUX, P. Ecology. New York: John Willey \& Sons, 1993. $688 \mathrm{p}$.

COOPS, H.; DOEF, R. W. Submerged vegetation development in two shallow, eutrophic lakes. Hydrobiologia, v. 340, p. 115-120, 1996.

DELARIVA, R. L. et al. Ichthyofauna associated to aquatic macrophytes in the Upper Paraná River floodplain. R. UNIMAR, supl.3, p. 41-60, 1994.

DELARIVA, R. L.; AGOSTINHO, A. A. Introdução de espécies: uma síntese. Acta Sci., v. 21, n. 2, p. 255-262, 1999.

DIBBLE, E. D.; KILLGORE, K. J.; HARREL, S. L. Assessment of fish-plant interactions. Am. Fish. Soc. Symp., v. 16, p. 357-372, 1996.

DUARTE, C. M.; KALFF, J. Littoral slope as a predictor of the maximum biomass of submerged macrophyte communities. Limnol. Oceanogr., v. 31, p. 1072-1080, 1986.

DUARTE, C. M.; KALFF, J.; PETERS, R. H. Patterns in biomass cover of aquatic macrophytes in lakes. Can. J. Fish. Aquatic. Sci., v. 43, p. 1900-1908, 1986.

DUARTE, C. M.; PLANAS, D.; PEÑUELAS, J.

Macrophytes, taking control of an ancestral home. In: MARGALEF, R. Limnology now: a paradigm of planetary problems. Amsterdam: Elsevier, 1994. p. 59-79.

DUTARTRE A.; HAURY J.; JIGOREL, A. A succession of Egeria densa in a drinking water reservoir in Morbihan (France). Hydrobiologia, v. 415, p. 243-247, 1999.

ENGELHARDT, K. A. M.; RITCHIE, M. E. Effects of macrophyte species richness on wetland ecosystem functioning and services. Nature, v. 411, p. 687-689, 2001.

ESTEVES, F. A.; CAMARGO, A. F. M. Sobre o papel das macrófitas aquáticas na estocagem e ciclagem de nutrientes. Acta Limnol. Bras., v. 1, p. 273-298, 1986. 
ESTEVES, F. A. Fundamentos de limnologia. Rio de Janeiro: FINEP/Interciência, 1998. 575 p.

FEIJOÓ, S. C. et al. Factors influencing biomass and nutrient content of the submersed macrophyte Egeria densa Planch., in a pampasic stream. Hydrobiologia, v. 341, p. 21-26, 1996.

FORD, D. E. Reservoir transport processes In: Thornton, K. W.; Kimmel, B. L.; Payne, F. E. Reservoir limnology: ecological perpectives. New York: John Wiley \& Sons, 1990. p. $15-41$.

FRENCH, T. D.; CHAMBERS, P. A. Habitat partitioning in riverine macrophyte communities. Fresh. Biol., v. 36, p. 509-520, 1996.

GOPAL, B. Water hyacinth. Amsterdam: Elsevier, 1987. $471 \mathrm{p}$.

HAHN, N. S. et al. Aspectos da alimentação do armado (Pterodoras granulosus)(Ostariophysi, Doradidae) em distintos ambientes do alto rio Paraná. R. UNIMAR, v. 14, p. 163-176, 1992.

HAKANSON, L.; PETERS, R. H. Predictive limnology. Methods for predictive modelling. Amsterdam, SPB Academic Publishing, 1995. 464 p.

HARLEY, K. L. S.; FORNO, I. W. Biological control of aquatic weeds by means of arthropods. In: PETERSE, A. H.; MURPHY, K. J. (Eds.) Aquatic weeds. The ecology and management of nuisance aquatic vegetation. New York: Oxford Science Publications, 1990. p. 177-185.

HARAMOTO, T.; IKUSIMA, I. Life cycle of Egeria densa Planch., na aquatic plant naturalized in Japan. Aquatic. Bot., v. 30, p. 389-403, 1988.

HOOTSMANS, M. J. M. Modelling Potamogeton pectinatus for better or for worse. In: MONTEIRO, A.; VASCONCELOS, T.; CATARINO, L. Management and ecology of aquatic plants. In: EWRS INTERNATIONAL SYMPOSIUM ON AQUATIC WEEDS, 1988, Lisbon. Proceedings... Lisbon: APRH, 1998. p.75-78.

HOOTSMANS, M. J. M. et al. Photosynthetic plasticity in Potamogeton pectinatus L. from Argentina: strategies to survive adverse light conditions. Hydrobiologia, v. 340, p. $1-5,1996$.

HOWARD-WILLIANS, C. et al. Macrophyte invasions. In: VINER, A. B. (Ed.). Inland waters of New Zealand. New Zealand: DSIR, 1987. (DSIR Bulletin, 241)

ITAIPU BINACIONAL. Ocorrência de plantas aquáticas em reservatórios de usinas hidrelétricas. Foz do Iguaçu: Itaipu Binacional, 1997. 9 p. (Relatório).
JANES, R. A.; EATON, J. W.; HARDWICK, K. The effects of floating mats of Azolla filiculoides Lam. And Lemna minuta Kunth on the growth of submerged macrophytes. Hydrobiologia, v. 340, p. 23-26, 1996.

JEPPESEN, E. et al. Top-down control in freshwater lakes: the role of nutrient state, submerged macrophytes and water depth. Hydrobiologia, v. 342/343, p. 151-164, 1997.

KRAULEC, F.; KAPLAN, Z. Diversity of Potamogeton species during 21 years of succession in a new water reservoir. Prestia, v. 66, p. 237-241, 1994.

KRÓLIKOWSKA, J. Eutrophication process in a shallow, macrophyte-dominated lake - species differentiation, biomass and the distribution of submerged macrophytes in Lake Luknajno (Poland). Hydrobiologia, v. 342/343, p. 411-416, 1997.

MANSOR, M. Noxious floating weeds of Malaysia. Hydrobiologia, v. 340, p. 121-125, 1996.

MEIJER, M. L.; HOSPER, H. Effects of biomanipulation in the large and shallow Lake Wolderwijd, The Netherlands. Hydrobiologia, v. 342/343, p. 335-349, 1997.

MITCHELL, D. S.; PIETERSE, A. H.; MURPHY, K. J. Aquatic plant problems and management in Africa. In: PIETERSE, A.H.; MURPHY, K. J. Aquatic weeds. New York: Oxford Science Publications, 1990. p. 341-354.

MURPHY, K. J.; BARRETT, P. R. F. Chemical control of aquatic weeds. In: PETERSE, A.H.; MURPHY, K. J.

Aquatic weeds. The ecology and management of nuisance aquatic vegetation. New York: Oxford Science Publications, 1990. p. 136-173.

NAKATANI, K.; BAUMGARTNER, G.; CAVICCHIOLI, M. Ecologia de ovos e larvas de peixes. In: VAZZOLER, A. E. A. M.; AGOSTINHO, A. A.; HAHN, N. S. A planície de inundação do alto rio Paraná: aspectos físicos, biológicos e socioeconômicos. Maringá: EDUEM/Nupélia, 1997. p. 281-306.

PIETERSE, A. H.; MURPHY, K. J. Aquatic weeds. New York: Oxford Science Publications, 1990. 593 p.

PIETERSE, A. H. The dramatic advance of water hyacinth (Eichhornia crassipes) in Africa in recent decades and options for its management: is further spread inevetable? In: MONTEIRO, A.; VASCONCELOS, T.; CATARINO, L. Management and ecology of aquatic plants. In: EWRS INTERNATIONAL SYMPOSIUM ON AQUATIC WEEDS, 10, 1998, Lisbon. Proceedings... Lisbon: APRH, 1998. p.279-282.

RIGLER, F. H.; PETERS, R. Science and limnology. Oldendorf: Ecology Institute, 1995. 239 p. 
ROONEY, N.; KALFF, J. Inter-annual variation in submerged macrophyte community biomass and distribution: the influence of temperature and lake morphometry. Aquat. Bot., v. 68, p. 321-335, 2000.

RØRSLETT, B.; JOHANSEN, S. Remedial measures connected with aquatic macrophytes in Norwegian regulated rivers and reservoirs. Regul. Rivers Res. Manag., v. 12, p. 509-522, 1996.

SAND-JENSEN, K. Influence of submerged macrophytes on sediment composition and near-bed flow in lowland streams. Fresh. Biol., v. 39, n. 4, p. 663-679, 1998.

SARBU, A.; CRISTOFOR, S. High competitive submeged macrophytes in the lower Danube floodplain and desta. In: MONTEIRO, A.; VASCONCELOS, T.; CATARINO, L. Management and ecology of aquatic plants. In: EWRS INTERNATIONAL SYMPOSIUM ON AQUATIC WEEDS, 10, 1998, Proceedings... Lisbon: APRH, 1998. p.119-122.

SCHEFFER, M. et al. Alternative equilibria in shallow lakes. Trends Ecol. Evol., v. 8, p. 276-279, 1993.

SOUZA, D. Padrões de diversidade alfa e beta de macrófitas aquáticas em diferentes escalas espaciais no reservatório de Itaipu. Maringá: Universidade Estadual de Maringá, 2000. 37 p. Dissertação (Mestrado em Ecologia) Universidade Estadual de Maringá, 2000.

SPENCE, D. H. N. The zonation of plants in freshwater lakes. In: MacFADYEN, A.; FORD, E. D. Advances in ecological research. London: Academic Press, 1982. p.37125 .

STANSFIELD, J. H. et al. Submerged macrophytes as refuges for grazing Cladocera against fish predation: observations on seasonal changes in relation to macrophyte cover and predation pressure. Hydrobiologia, v. 342/343, p. 229-240, 1997.

TAVECHIO, W. L. G.; THOMAZ, S. M. Effects of light on the growth and photosynthesis of Egeria najas Planchon. Braz. Arch. Biol.Technol. (no prelo).

THOMAZ, S. M.; BINI, L. M. Ecologia e manejo de macrófitas em reservatórios. Acta Limnol. Brasil., v. 10, n. 1, p. 103-116, 1999a.
THOMAZ, S. M.; BINI, L. M. A expansão das macrófitas aquáticas e implicações para o manejo de reservatórios: um estudo na represa de Itaipu. In: HENRY, R. Ecologia de reservatórios: estrutura, função e aspectos sociais. Botucatu: Fundibio, 1999b. p. 599-625.

THOMAZ, S. M.; BINI, L. M.; SOUZA, D. C. Biomass and maximum colonization depth of Egeria najas Planchon (Hydrocharitaceae) at Itaipu Reservoir, Brazil. In: MONTEIRO, A.; VASCONCELOS, T.; CATARINO, L. Management and ecology of aquatic plants. In: EWRS INTERNATIONAL SYMPOSIUM ON AQUATIC WEEDS, 10, 1998, Lisbon. Proceedings... Lisbon: APRH, 1998. p223-226.

THOMAZ, S.M. et al. Aquatic macrophytes of Itaipu reservoir, Brazil: Survey of species and ecological considerations. Braz. Arch. Biol. Technol., v. 42, n. 1, p. 15-22, 1999.

TUNDISI, J. G. Tropical South America: Present and perspectives. In: MARGALEF, R. Limnology now: a paradigm of planetary problems. Amsterdam: Elsevier, 1994. p. $353-424$.

VOLTZ, J. Reservoirs and nature - a contradiction? J. Water Suppl. Res. Technol., v. 44, p. 30-34, 1995.

WALLSTEN, M. The shallow lake Hjälstaviken - aquatic vegetation and waterfowl. In: Monteiro, A.; Vasconcelos, T.; Catarino, L. Management and ecology of aquatic plants. In: EWRS INTERNATIONAL SYMPOSIUM ON AQUATIC WEEDS, 10, 1998 Lisbon. Proceedings... Lisbon: APRH, 1998. p. 151-154.

WEAVER, M. J.; MAGNUSON, J. J.; CLAYTON, M. K. Distribution of littoram fishes in structurally complex macrophytes. Can. J. Fish. Aquatic. Sci., v. 54, p. 2277 2289, 1997.

WETZEL, R. G. Limnology. Fort Worth: Saunders College Publishing, 1983. 767 p.

WINTON, M. D.; CLAYTON, J. S. The impact of invasive submerged weed species on seed banks in lake sediments.

Aquatic. Bot., v. 53, p. 31-45, 1996. 